\title{
LIMITATIONS OF VESTIBULAR REHABILITATION THERAPY USING DYNAMIC COMPUTERIZED POSTUROGRAPHY IN PATIENTS WITH CENTRAL VESTIBULAR SYNDROME
}

\author{
Anamaria Andreia Ulmeanu , Andreea Didilescu², Raluca Enache ${ }^{3}$, Gabriela Musat ${ }^{2}$, \\ Codrut Sarafoleanu ${ }^{2}$ \\ ${ }^{1}$ Bucharest Emergency Hospital \\ 2 "Carol Davila" University of Medicine and Pharmacy, Bucharest \\ ${ }^{3}$ Sarafoleanu Medical ENT Clinic, Bucharest
}

\begin{abstract}
Objectives. To evaluate the importance of computerized dynamic posturography in vestibular rehabilitation (VR) of patients with central vestibular syndrome.

Methods. The study included 30 patients with central vestibular syndrome with mean age $( \pm S D)=72.96 \pm 11.97$ which benefited from VR on a posturography platform between 2012-2014. All patients were evaluated using sensory integration tests. The parameters studied were: Romberg coefficient, statokinesigram (SKG), maximum amplitude of the degree of deviation in anterior-posterior and medial-lateral planes, SKG and the time interval of the rehabilitation program.

Results. All of the analyzed parameters showed statistically significant results $(p<0.05)$. The anterior-posterior and medio-lateral balance improved significantly at the end of the vestibular rehabilitation program and the parameters decreased to values close to normal.

Conclusions. For patients with central vestibular syndrome, the vestibular rehabilitation improves postural stability and quality of life by reducing the risk of falls.
\end{abstract}

Keywords: posturography, central vestibular syndrome, vestibular rehabilitation, risk of fall

\section{INTRODUCTION}

Computerized Dynamic Posturography gives an objective assessment of the overall balance of the patient and also highlights what system uses each subject for maintaining posture. (1) Vestibular rehabilitation on a posturography platform is achieved by the selecting games runned in different sequences, depending on the condition that causes impaired balance and aims at the recovery of the vestibular function. $(2,3)$ The purpose of this study was to determine the value of vestibular rehabilitation performed using a posturography platform in patients with central vestibular deficit.

\section{MATERIAL AND METHODS}

The study included 30 patients with central vestibular syndrome who received vestibular rehabilitation using platform posturography between 20122014. The patients who participated in this study signed an informed consent form and all patients have completed the whole vestibular rehabilitation program. The study included patients diagnosed with central vestibular deficit and who have never benefited from vestibular rehabilitation on platform posturography. We excluded the patients who refused inclusion in the study, those with associated neurological disorders (dementia, Parkinson's, etc.),

Author for correspondence:

Anamaria Andreia UImeanu, Emergency Hospital, 169 Splaiul Independentei, Bucharest

E-mail: andreia21@gmail.com 
psychiatric disorders, visual disturbances, patients treated with neuroleptics, sedatives, antiepileptics, antidepressants, drugs or alcohol abuse, patients with severe orthopedic dysfunction and arthrosis of the ankle, hip and knee.

\section{STUDY PROTOCOL}

For this research we took into account the results obtained from patients that were tested with eyes open (EO) and eyes closed (EC) on foam platform. Subjects were evaluated using sensory integration tests. We used Posturographic Synapsys System (version 3.0, SYNAPSYS, Marseille, France). The evaluation of patients was performed before and after the eight sessions of vestibular rehabilitation. The tests consisted of two trials each of 20 seconds. The VR program consisted of games included in the software used by the posturographic system. Each session began with a test reference, which helped monitor the patient's evolution throughout the rehabilitation. The same game benchmark was used for each session separately and for each patient included in the study. Four game groups were included in the rehabilitation session: Stabilization, Weight shift, Weight bearing and Postural control.(3)

\section{STATISTICAL ANALYSIS}

To evaluate the success of VR therapy on posturography platform, we created two groups of patients, according to their performance: improved and stationary. For these two groups we defined limit values for each parameter included in our research, both for eyes open and eyes closed condition on foam platform. These are shown in Table 1.

TABLE 1. Representation of range values for improved and stationary group

\begin{tabular}{|l|c|c|}
\hline FOAM & IMPROVED & STATIONARY \\
\hline SKG EO $\left(\mathrm{mm}^{2}\right)$ & $618-900$ & $>900$ \\
\hline$A P(\mathrm{~mm})$ & $38-50$ & $>50$ \\
\hline $\mathrm{ML}(\mathrm{mm})$ & $40-52$ & $>52$ \\
\hline $\mathrm{SKG} \mathrm{EC}\left(\mathrm{mm}^{2}\right)$ & $1325-2200$ & $>2200$ \\
\hline $\mathrm{AP}(\mathrm{mm})$ & $67-80$ & $>80$ \\
\hline $\mathrm{ML}(\mathrm{mm})$ & $58-70$ & $>70$ \\
\hline
\end{tabular}

The collected data were analyzed using the software StataIC 11 (StataCorp. 2009. Stata: Release 11 Statistical Software. College Station, TX, USA). The data were expressed as a percentage, frequency, mean, standard deviation, depending on the situation. T test (Student) was used for the eva- luation of quantitative differences in the calculation of media. The level of statistical significance was set at 0.05 .

\section{RESULTS}

The study was conducted on a group of 30 patients with central vestibular syndrome, consisting of 19 women (63.3\%) and 11 men (36.67\%); 13 cases from rural areas $(43.33 \%)$ and 17 cases from urban areas $(56.67 \%)$. The average age of was 72.9 years $\pm 11.97 \mathrm{SD}$ (aged between 37 and 90 years).

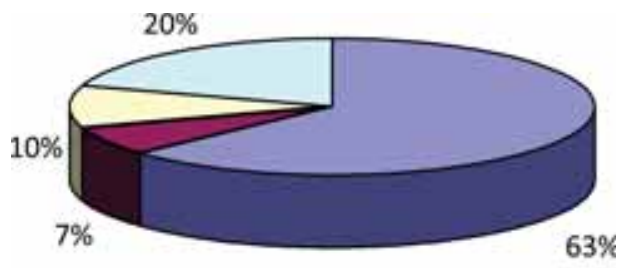

口Unknown etiology of CVS DMultiple sclerosis

- Vestibular omissions $\quad$ V Vestibular migraine

CHART 1. Distribution of cases according to etiology

For the patients included in this study $20 \%$ were diagnosed with vestibular omissions, $10 \%$ with multiple sclerosis and $7 \%$ of with vestibular migraine. In $63 \%$ of these cases, the cause for the central vestibular deficit was unknown, they showed demyelination and areas of cerebral atrophy on the brain MRI.

The statistical analysis of the associated risk factors, revealed mostly cervical spondylosis in 15 cases $(50 \%)$. The second risk factor was essential hypertension with 14 cases $(46.67 \%)$, followed by diabetes with 10 cases $(33.33 \%)$, carotid atheromatosis and dyslipidemia with 8 cases each representing $26.67 \%$. The remaining patients in the study were diagnosed with stroke in 3 cases accounting for $10 \%$. All parameters studied showed statistically significant results $(\mathrm{p}<0.05)$. (Table 2 )

Romberg coefficient, statokinesigram (SKG), maximum amplitude of the degree of deviation in anterior/posterior and medial/lateral planes, SKG and the time interval of the rehabilitation program

RQ1 and RQ2: Romberg coefficient before and after RV; SKG1 and SKG2 foam EO: statokinesigram with eyes open before and after VR on foam platform; AP1 and AP2 foam EO: maximum amplitude of the degree of deviation with eyes open in the anterior-posterior plane before and after VR on foam platform; ML1 and ML2 foam EO: maximum amplitude of the degree of deviation with eyes open in medio-lateral plane before and after VR on foam platform; SKG1 and SKG2 foam EC: statokinesig- 
TABLE 2. Correlations of mean values of the studied parameters

\begin{tabular}{|c|c|c|c|c|c|}
\hline Variables & Media & $\begin{array}{l}\text { Standard } \\
\text { Deviation }\end{array}$ & Min. & Max. & p \\
\hline RQ1 & 278,93 & 176,75 & 57 & 850 & \multirow[t]{2}{*}{$p=0,0077$} \\
\hline RQ2 & 200,56 & 89,15 & 66 & 460 & \\
\hline SKG1 foam EO & 1484,36 & 1374,27 & 436,49 & 8165,22 & \multirow[t]{2}{*}{$p=0,0057$} \\
\hline SKG2 foam EO & 925,99 & 499,05 & 360,64 & 2959,51 & \\
\hline AP1 foam EO & 45,5 & 13,94 & 27,6 & 95 & \multirow[t]{2}{*}{$p=0,0018$} \\
\hline AP2 foam EO & 37,64 & 6,5 & 23,9 & 55,1 & \\
\hline ML1 foam EO & 50,37 & 16,38 & 30 & 118,2 & \multirow[t]{2}{*}{$p<0,0001$} \\
\hline ML2 foam EO & 39,04 & 9,05 & 28,4 & 72,2 & \\
\hline SKG1 foam EC & 4254,95 & 2736,05 & 1813,08 & $15.915,73$ & \multirow[t]{2}{*}{$p=0,0014$} \\
\hline SKG2 foam EC & 2739,71 & 951,55 & 757,43 & 4940 & \\
\hline AP1 foam EC & 86,68 & 22,02 & 46,7 & 146,7 & \multirow[t]{2}{*}{$p<0,0001$} \\
\hline AP2 foam EC & 68,98 & 19,12 & 7,2 & 117,2 & \\
\hline ML1 foam EC & 80,53 & 22,43 & 49,7 & 136,4 & \multirow[t]{2}{*}{$p=0,0002$} \\
\hline ML2 foam EC & 66,53 & 18,07 & 35,2 & 112 & \\
\hline SKG1 before VR & 32,53 & 12,49 & 8 & 48 & \multirow[t]{2}{*}{$p<0,0001$} \\
\hline SKG2 after VR & 24,56 & 10,59 & 3 & 42 & \\
\hline T1 before VR & 25,03 & 9,37 & 10,05 & 38,86 & \multirow[t]{2}{*}{$p<0,0001$} \\
\hline T2 after VR & 20,03 & 7,11 & 8,09 & 28,86 & \\
\hline
\end{tabular}

ram with eyes closed before and after VR on foam platform; AP1 and AP2 foam EC: maximum amplitude of the degree of deviation with eyes closed in the anterior-posterior plane before and after VR on foam platform; ML1 and ML2 foam EC: maximum amplitude of the degree of deviation with eyes closed in medio-lateral plane before and after VR on foam platform; SKG1 and SKG2 before and after rehabilitation program: statokinesigram of the reference game before and after vestibular rehabilitation process; $\mathrm{T} 1$ and $\mathrm{T} 2$ before and after rehabilitation program: the time interval (seconds) of the reference game before and after vestibular rehabilitation process.

\section{Statistical analysis of parameters with EO and EC on foam platform before and after vestibular rehabilitation on platform posturography}

In Eyes Open condition, there was a significant decrease of the statokinesigram after VR $(p<0.05)$ from an average of 1,484.36 $\pm 1,374,27 \mathrm{~mm}$ before VR to an average of $925.99 \pm 499,05 \mathrm{~mm}$ after VR. The average difference was $558.36(\mathrm{p}=0.0057$; Student t-test; IC 95\% is 76.3 to 940.4 ). After the rehabilitation we observed a decrease in balance on AP plan $(\mathrm{p}<0.05)$ from a mean of $45.5 \pm 13,94 \mathrm{~mm}$ to an average value of $37.64 \pm 6.5 \mathrm{~mm}$. The average difference is $7.86(\mathrm{p}=0.0018$; Student t-test; IC $95 \%$ is 3.19 to 12.52 ). Regarding balance on ML plan after VR, the results show a significant decrease $(\mathrm{p}<0.05)$ from $50.37 \pm 39.04$ to $16,38 \mathrm{~mm} \pm$ $9,05 \mathrm{~mm}$. The average difference is $11.32(\mathrm{p}$ $<0.0001$; Student t-test; IC $95 \%$ is 6.84 to 15.8 ).
In Eyes Closed condition, there is a significant decrease of the statokinesigram after VR $(\mathrm{p}<0.05)$ from an average of 4,254.95 $\pm 2,736,05 \mathrm{~mm}$ before VR by an average of of 2,739.71 $\pm 951,55 \mathrm{~mm}$ after VR. The average difference is $1,515.23(\mathrm{p}=0.0014$; Student t-test; IC $95 \%$ is 636.8 to $2,393.66)$. The balance on AP plan improves significantly $(\mathrm{p}<0.05)$ after VR from an average of $86.68 \pm 22.02$ to 68.98 $\pm 19,12 \mathrm{~mm}$. The average difference is 17.69 (p $<0.0001$; Student t-test; IC $95 \%$ is 16.8 to 31.3 ). ML balance after VR presents a significant decrease $(\mathrm{p}<0.05)$ from $80.53 \pm 22.43 \mathrm{~mm}$ to an average of $66.53 \pm 18,07 \mathrm{~mm}$. The average difference is $14(\mathrm{p}$ $=0.0002$; Student t-test; IC $95 \%$ is 7.41 to 20.59 ).

\section{Statistical analysis of statokinesigram $\left(\mathrm{cm}^{2}\right)$ and time (seconds) of the reference game before and after the vestibular rehabilitation process}

The time interval of the reference game is reduced significantly $(\mathrm{p}<0.05)$ after VR, from a mean of $25.03 \pm 9,37 \mathrm{~s}$ before VR to an average of 20.03 $\pm 7,11 \mathrm{~s}$ after VR. The average difference is $5(\mathrm{p}$ $<0.0001$; Student t-test; IC $95 \%$ is 3.37 to 6.63 ). In terms of the SKG obtained in the reference game after VR, there was a significant decrease $(\mathrm{p}<0.05)$ from an average of $32.53 \pm 12,49 \mathrm{~cm}^{2}$ before VR to an average of $24.56 \pm 10,59 \mathrm{~cm}^{2}$ after VR. The average difference is $7.96(\mathrm{p}<0.0001$; Student t-test; IC $95 \%$ is 5.56 to 10.36). All parameters studied showed highly statistically significant results $(p$ $<0.05$ ) at the end of the 8 sessions of vestibular rehabilitation. 


\section{DISCUSSION}

Final evaluations showed that from the group of 30 patients with central vestibular syndrome who benefited from vestibular rehabilitation on posturography platform, only 12 patients showed a significant improvement in evolution (40\%) and 14 patients $(46.67 \%)$ had a stationary evolution. Most satisfactory results were recorded in 4 cases $(13.33 \%)$, where the evolution of the subjects was very good at the completion of the first meeting of vestibular rehabilitation. In only $13.33 \%$ of the cases full recovery of vestibular function was achieved. The recovery capacity depends very much on the vestibular function deficit of the affected vestibular organ. Patients with central vestibular syndromes, may have the adaptation and compensation functions affected or they can have a progressive disease. Thus, improving postural control is often more difficult than in patients with peripheral vestibular syndrome. There are studies showing that by controlled and monitored rehabilitation programs we can obtain a high level of recovery in comparison with the cases that remain untreated. $(4,5)$

Aging and/or associated risk factors such as diabetes, hypertension, and stroke, influence balance and posture by increasing instability and the risk of falls and for that reason improvement of postural control is made more difficult. $(6,7)$ For patients diagnosed with central vestibular deficit, vestibular rehabilitation on posturography platform involves improving postural stability and quality of life by reducing the risk of falls, improvement of neuromuscular coordination and decrease of anxiety due to vestibular disease. $(8,9)$ The final results obtained during eyes open condition on foam platform show that the maximum amplitude values of the anterior-posterior and medio-lateral balance decreased significantly $(p<0.05)$ into the normal range of values. The statokinesigram obtained in the same terms, also shows a significant decrease ( $p$ $=0.0057)$. Similar results were recorded during eyes closed condition $(\mathrm{p}<0.05)$ for AP and ML oscillations and for SKG $(p<0.0014)$. The results of this study indicate that the use of VR with eyes closed and eyes open on platform posturography leads to an improvent of balance and neuromuscular coordination in patients with central vestibular syndrome patients, and are consistent with information in the literature. Badke showed in a retrospective study conducted in 2005 on 32 patients (12 with peripheral vestibular deficiency and 20 with central vestibular deficit and mixed) an important improvement of balance and quality of life after vestibular rehabilitation. (5) Also, Rossi-Izquierdo et al evaluated the results of the vestibular rehabilitation on platform posturography in patients with central vestibular deficit. (8) From 45 patients with Parkinson's disease, VR on platform posturography was performed in 10 cases that presented a high risk of falling. At the end of therapy the authors noted a significant improvement of scores for Sensory Integration Test with an increase in stability and quality of life, as well as decrease of the risk of falls. (8) Similar results were seen during our study.

Statistical analysis of the statokinesigram and the time interval of the reference game before and after the RV were found to be significant $(p<0.0001)$ in terms of assessing therapeutic success of RV in patients included in the study.

\section{CONCLUSIONS}

A proper posture is essential for the effective functioning of the human body. In patients with central vestibular syndrome, vestibular rehabilitation implies improving postural stability and quality of life by reducing the risk of falls. Successful treatment by vestibular rehabilitation requires active patient cooperation and participation inducing a positive psychological effect with an impact on self confidence and improving physical condition, thus improving quality of life. Vestibular rehabilitation programs on platform posturography in patients with central vestibular deficit encompasses all these advantages and allows evaluation and monitoring of medium and long-term performance.

\section{REFERENCES}

1. Georgescu M. Evaluation of dizzy pacient, Ed. Maiko, Bucharest 2005. Chapter VII: p. 134-135.

2. Krebs, D.E., et al., Double-blind, placebo-controlled trial of rehabilitation for bilateral vestibular hypofunction: preliminary report. Otolaryngol Head Neck Surg, 1993. 109(4): p. 735-41.

3. Synapsys posturography system, User manual, version 3.0 Rev C

4. Black F.O., et al. Outcomes analysis of individualized vestibular rehabilitation protocols. Am J Otol. 2000; 21(4):543-51

5. Badke M.B., Miedaner J.A., Shea T.A., Grove C.R., Pyle G.M. Effects of vestibular and balance rehabilitatio on sensory organization 
and dizziness handica. The Annals of Otology, rhinology, and laryngology, 2005; 114:48-54

6. H. Suarez, M. Arocena, A. Suarez, T.A. DE Artagaveyti, P. Muse, J. GIL. Changes in Postural Control Parameters after Vestibular Rehabilitation in Patients with Central Vestibular Disorders. Acta Otolaryngol 2003; 123: 143-147

7. Peterka R.J., Black F.O., Schoenhoff M.B. Age related changes in human vestibulo-ocular and optokinetic reflexes: pseudorandom rotation and caloric test. J Vestib Res 1990; 1: 49-59

8. Rossi-Izquierdo M., Soto-Varela A., Santos-Perez S, et al. Vestibular rehabilitation with computerised dynamic posturography in patients with Parkinson's disease: improving balance impairment. Disab Rehabil. 2009; 31(23):1907-16.

9. Musat G. Computerized posturgraphy in the diagnosis and treatment of te instability of the elderly. Romanian Journal of Neurology, vol XII, No 2, 2013; p.58-62

10. H. Suarez, M. Arocena, A. Suarez, T.A. DE Artagaveyti, P. Muse and J. GIL. Changes in Postural Control Parameters after Vestibular Rehabilitation inPatients with Central Vestibular Disorders. Acta Otolaryngol 2003; 123: 143-147 